\title{
Elderly patient: which vascular access? Choice and management of vascular access in the elderly patient
}

Nephrology@ Point of Care Volume 4: I-5

(C) The Author(s) 2018 Reprints and permissions: sagepub.co.uk/journalsPermissions.nav DOI: 10.1 1 77/20593007/8755625 journals.sagepub.com/home/npc

(S)AGE

\author{
Mirko Menegolo', Andrea Xodo', Marianna Alessi², Carlo Maturi', \\ Francesca Simioni², Barbara Rossi², Lorenzo Antonio Calò'², \\ Michele Antonello' and Franco Grego'
}

\begin{abstract}
The optimal vascular access for elderly patients remains a challenge due to the difficult balance between risks and benefits in a population with increased comorbidity and decreased survival. Long dependence to central vein catheter, patient comorbidities, life expectancy, and complication rates are important influencing the indications for arteriovenous fistula or an arteriovenous graft. Although central vein catheters are simpler way to start a hemodialysis treatment, elderly patients are also at higher risk of death from infection or other complications associated with them more than for younger patients. The discussion revolves around the following key questions:

I. What are the limiting factors for a vascular access in the elderly patients?

2. Central venous catheter-is it still an option for elderly patients?

3. Is still the autologous arteriovenous fistula playing a pivotal role as hemodialysis access in the elderly patients?

4. Are there any real surgical contraindication to perform a vascular access in elderly patients?

5. Is the old age a limiting factor for the vascular access management?
\end{abstract}

\section{Keywords}

Arteriovenous fistula, arteriovenous graft, early cannulation graft, vascular access, elderly patients, hemodialysis.

Date received: 30 November 20I7; accepted: 8 January 2018

\section{Case report}

An 80-year-old woman presented with a history of hypertension, chronic obstructive pulmonary disease (COPD), peripheral vascular disease, and chronic renal failure (stage 5, estimated glomerular filtration rate (eGFR): $12.6 \mathrm{~mL} / \mathrm{min} / 1.73 \mathrm{~m}^{2}$ ), previously treated for abdominal aortic aneurysm by endovascular aneurysm repair (EVAR).

The patient was regularly followed at the nephrology clinic and was evaluated by the vascular surgeon for the placement of a vascular access (VA), in preparation for dialysis treatment. The duplex scan examination showed postphlebitic fibrosis of the cephalic veins in both arms and a small size of both basilic veins $(2.5 \mathrm{~mm}$ diameter $)$. In this case, given the poorness of the autologous superficial venous system, the patient was treated through a prosthetical left omero-axillary arteriovenous bypass. The procedure was performed in local anesthesia (LA) using a Gore Hybrid $^{\circledR}$ (W. L. Gore \& Associates, Inc., Flagstaff, AZ, USA) vascular graft. This choice was done since the patient did not need an immediate ultrafiltration (whereby an early

\footnotetext{
'Division of Vascular and Endovascular Surgery, Department of Cardiac, Thoracic and Vascular Sciences, School of Medicine, University of Padova, Padova, Italy

2Division of Nephrology, Department of Medicine, School of Medicine, University of Padova, Padova, Italy

Corresponding author:

Mirko Menegolo, Division of Vascular and Endovascular Surgery, Department of Cardiac, Thoracic and Vascular Sciences, School of Medicine, University of Padova, Via Giustiniani 2, 35121 Padova, Italy. Email: mirko.menegolo@unipd.it
} 
cannulation graft, which can be cannulated within $24 \mathrm{~h}$ of implantation, is preferred) and also because this graft allows a fast operation, thanks to the sutureless venous extremity. The patient started dialysis 2 months later and the follow-up consisted in a duplex scan evaluation every 3 months postimplantation. At the present time, 7 months later, the graft is patent, no signs of venous stenosis have been detected, and the patient is successfully treated through a tri-weekly dialysis.

\section{Introduction}

In recent years, the demography of the global population is changing: people over the age of 65 years have increased, especially in Western countries, and in 2050, the percentage of the elderly population probably will exceed two billions, with consequent implications for health and care. ${ }^{1}$ Old age is distinguished for at least two major groups: the elderly (60-75 years) and the very elderly (over 76 years) patients. The high prevalence of chronic kidney disease (CKD) in the elderly is attributable not only to the growing prevalence of traditional risk factors such as diabetes, hypertension, and cardiovascular diseases but also to the new CKD definition criteria, based on the eGFR and the presence of markers of renal damage (proteinuria, radiological alterations, urinary sediment, and cellular biopsy alterations). ${ }^{2}$ At the present time, there are no specific guidelines and recommendations regarding early identification and management of elderly with CKD. The major goal of a screening program should be to identify elderly with CKD at an early stage, in order to prevent cardiovascular events and/or progression to end-stage renal disease (ESRD). It is well known that early referral to nephrologists has been associated with decreased mortality, better outcome, and cost saving; furthermore, pharmaceutical and surgical innovations and lifestyle modifications in elderly population with CKD may have a favorable effect on patients' outcomes. ${ }^{3}$ Older people today are candidates for a large number of procedures and interventions that have not been considered for them in the past, including kidney replacement therapy (dialysis and transplantation). Today, there is a dilemma whether it is proper to use an expensive treatment such as hemodialysis (HD) which charges all the national health systems or to prefer a non-dialytic management in a growing subpopulation with a limited life expectancy: ${ }^{4}$ for many nephrologists, delaying the onset of dialysis in association with a protein restriction could be an alternative strategy. ${ }^{5}$ Regarding the elderly with ESRD (eGFR $<10 \mathrm{~mL} / \mathrm{min}$ ), there are conflicting data about survival among patients undergoing dialysis versus those receiving non-dialytic management. In the United States, the 1-year survival rate of patients over 80 years after dialysis initiation is above $54 \%$, whereas mortality rate is high (above 20\%) during the first 3 months, probably due to underlying illness and to the significant comorbidities. ${ }^{6}$
Another aspect to be considered is related to the cost of public health: preemptive arteriovenous fistula (AVF) placement may not be the cost-effective approach in older adults compared with the option to place an arteriovenous graft (AVG); the AVF option shows cost savings for younger patients and for those with longer life expectancy, while it does not seem to have cost savings for the patients $>80$ years. ${ }^{7}$ Given these considerations, the decision to treat the ESRD in the elderly provides a fair balance of risks and benefits and should be evaluated case by case.

\section{What are the limiting factors for a VA in the elderly patients?}

A well-functioning VA is crucial for a good HD. Elderly patients who are candidated to HD are usually affected by numerous comorbidities (such as diabetes, micro/macroangiopathy, and cardiovascular disease) that can impair the outcome of a VA as well as be impaired by a creation of an arteriovenous shunt.

Chronic heart failure (CHF) is often present in these patients and can be reasonably aggravated by the creation of an AVF, even if it is not described as an absolute contraindication for a VA. However, patients in class New York Heart Association (NYHA) $\geq 2$ have been shown to have an improvement in CHF symptoms after VA closure. ${ }^{8}$ Despite this, CHF seems to have a little implication in VA in elderly patients: for those $>65$ years in $\mathrm{HD}$ and with a diagnosed CHF, the death rate from cardiac causes seems not to be higher in those with a fistula compared with the group with central venous catheter (CVC). ${ }^{9}$

Peripheral veins damaged by chemotherapy, vein puncture, or other trauma are particularly associated with poor AVF outcomes, while central vein injuries caused by central catheters, pacemakers, or other interventions may prohibit both AVF and AVG creation. In addition, elderly patients often come to the attention of the nephrologist shortly before the onset of renal replacement therapy (late referral), adversely affecting the VA outcomes. Poor peripheral artery conditions can unavoidably affect the outcome of a VA as well as an AV shunt can impair a preexisting peripheral artery disease. Another factor to be considered in setting up VA in the elderly is the "fragile elderly syndrome." The components of frailty encompass functional status and include weight loss, poor endurance, low energy, weakness and may manifest as slow walking speed and low physical activity: this is a relevant problem because up to $75 \%$ of patients on HD over 60 years meet the criteria for frailty. Having a CVC with a maturing AVF has been reported to be associated with a greater risk of mortality than AVG alone in patients with limited functional status. ${ }^{10}$ An additional important element to consider is the patient compliance, whether it is intradialytic (fixed position of the arm during HD), intraoperative for local or regional anesthesia (RA), and during the cure and follow-up of VA, 
which requires patients' collaboration. The low compliance of the elderly (patients "not fit for" LA or RA), along with upper extremities poor arteries and veins "heritage," may represent the main limitation for a VA construction.

\section{CVC—is it still an option for elderly patients?}

CVCs are associated with a significantly higher risk of infections, mechanical complications, thrombosis, and mortality: Bray et al. ${ }^{8}$ revealed a threefold increased risk in mortality (all causes) and a sevenfold increased risk in death from septicemia with the use of CVCs compared with patients using AVF or AVG. The presence of a CVC also appears to be an obstacle for a subsequent VA, especially for elderly: for Wasse et al., ${ }^{11}$ in fact, patients older than 75 years were more than twofold remaining CVC dependent at 90 days compared with those younger than 50 years.

The global costs for an AVF or an AVG, initially higher than those required for a $\mathrm{CVC}$, are amortized by the reduction in septic and post-operative complications, as well as hospitalization days, with a potential savings of $£ 1000$ for a 6-month treatment with an early cannulation AVG (ecAVG) compared to a CVC. ${ }^{9}$

Central vein obstructions are related, in the majority of patients, to a previous insertion of CVC: central venous obstruction develops in more than $40 \%$ patients with subclavian vein catheters and with a little less incidence in patients with jugular vein catheter. ${ }^{9}$ This is the most important cause of AVF or AVG failure, requiring further additional endovascular procedures in order to guarantee the central vein outflow. ${ }^{12}$ Even if CVC still has the advantage of being the least invasive procedure and the faster solution for a late referral patient, the clinical consequences that its stubborn use can have should not be underestimated.

The AVF creation is usually a mini-invasive procedure and can be performed in the majority of cases, including diabetics and old patients: age alone should not in fact disqualify patients from VA surgery since there is a high rate of technical feasibility for fistula construction.

RA and LA are usually the techniques of choice for AVF creation, while general anesthesia (GA) could represent a contraindication for AVF/AVG creation in the elderly patients, especially for peri- and post-operative implications related to the patients' comorbidities. ${ }^{13}$

\section{Is still the autologous AVF playing a pivotal role as HD access in the elderly patients?}

According to the current guidelines, the autologous AVF is the first and preferred VA for HD because of its long-term patency and the low incidence of infections. ${ }^{14,15}$ However, there are no universally recognized indications about the $\mathrm{HD}$ access in the elderly and especially in the very elderly patients: in the global ESRD population, one-third of AVF fails to mature, ${ }^{16}$ whereas in patients over 65 years, this risk is doubled. ${ }^{17}$

The cumulative patency of AVG has been reported to be superior to AVF in elderly patients: ${ }^{18}$ in contrast, in a study with patients older than 80 years, AVF patency is comparable to that of their younger counterparts. ${ }^{19}$

DeSilva et al. ${ }^{20}$ showed that patients aged 67-79years had higher survival from pre-dialysis placement of AVF compared with AVG, different from what is found in the octogenarians and nonagenarians: this could be explained by the greater number of elderly patients at risk of initiating HD with a CVC because of unsuccessful or delayed maturation of AVFs. In addition, the competitive risk of death in octogenarians is higher and may minimize the potential long-term benefits of placing an AVF over an AVG.

Cui et al. ${ }^{21}$ showed that AVG and AVF had similar primary and secondary patency rates in elderly patients but fistulas exhibit a much higher primary failure rate, requiring more interventions to achieve maturation and a longer period of catheter dependence. Elderly patients with ESRD have a higher prevalence of comorbidities, which increases their procedure-related risk. ${ }^{22}$ Therefore, AVG may be the preferred access option for select elderly patients because of its lower intervention rate to achieve maturation. Moreover, in late referral patients needing to start immediately HD, the use of last-generation AVG, which can be cannulated within $24 \mathrm{~h}$ of implantation, would allow the omission of temporary venous catheterization without affecting perioperative morbidity rates and long-term patency rates. Tozzi and Franchin's ${ }^{23}$ experience with Gore Acuseal (W. L. Gore \& Associates, Inc.) demonstrated good patency rates and low complication rates of this ecAVG. Aitken et al. compared two groups of patients receiving ecAVG and CVC. Bacteremia developed in $16.4 \%$ of patients in the CVC group compared with $3.3 \%$ in the ecAVG group and mortality was also higher for patients with CVC (16\%) compared to those with ecAVG (5\%). ${ }^{24}$ The cost of public health still offers some reflections: preemptive AVF placement may not be the cost-effective approach in older adults. Compared with the option to place an AVG, the AVF option shows cost savings for younger patients and for those with longer life expectancy and shows no longer cost savings for the patients $>80$ years, so AVF first is not clearly a superior strategy in the elderly population. ${ }^{7}$ Given the higher clinical risk of these patients and the short life expectancy, AVG may be a reasonable first-line choice in select elderly patients with inadequate superficial veins and especially in late referrals who require accelerated cannulation to avoid CVC placement. 


\section{Are there any real surgical contraindication to perform a VA in elderly patients?}

Vessel's quality is the first crucial point that could affect the result of a VA, especially in the elderly patients. Vessel mapping has been highly encouraged and current international guidelines support the routine use of color Doppler ultrasound (DUS) before VA surgery. Several anatomic parameters (artery diameter, calcifications, resistance index, arterial blood flow, vein diameter before and after proximal vein compression) may help the examinator to evaluate the vessel suitability and the best surgical solution for AVF creation. There are many factors that may interact to determine the success or failure of any individual AVF: a meta-analysis of 13 cohort studies showed that wrist radiocephalic AVF had an increased primary failure rate and reduced patency in elderly patients at all time points. ${ }^{25}$ In comparison with a younger cohort, elderly patients are more likely to have a poor-quality forearm or leg veins and are more likely to have atheroma or calcifications affecting their arm arteries. ${ }^{26}$ Women usually have smaller arteries and this may be the reason for poorer maturation and survival rates of VA for this group of patients, associated with a higher number of access revisions. Diabetes and concomitant arteriosclerosis may have an additional negative impact on the chance of successful access creation because these patients usually have thickened and calcified arteries with proximal or distal vessel obstructions. ${ }^{12}$

Small diameter arteries and veins are associated with a poor outcome of VA: in particular, cephalic vein diameter less than $3 \mathrm{~mm}$ can take a maturation time higher than 3 to 4 months and this cannot be accepted for elderly late referral patients. ${ }^{27}$

Venography and central vein evaluation should be performed in patients known to have a previous catheter or pacemaker/implantable cardioverter defibrillator (ICD). ${ }^{12,28}$ The finding of a central vein stenosis should not be considered as an absolute contraindication in case of no other options for VA since a concomitant endovascular procedure can be performed at the same time of the AVG implantation. Incidence of VA complications is higher in very elderly patients, and steal syndrome, commonly referred to as hand ischemia or "steal" after dialysis access placement, is a serious complication, with age greater than 65 years been recognized as a potential risk factor: this underlines again the role of preoperative vessels' investigation, in order to avoid the construction of a VA in patients with small or calcified arteries. ${ }^{29}$

Frailty and malnutrition, commonly seen in an elderly patient submitted to HD, often lead to thin skin and easy bruising tendencies that cause a relatively higher incidence of skin tears or hematomas; in addition, anticoagulant and anti-platelet drugs that are often used by these patients can also potentially increase the risk of bleeding complications.
Very elderly patients on HD are particularly fragile and often affected by multiple comorbidities: in particular, chronic diseases may coexist with geriatric syndromes like frailty, falls, insomnia, or dementia and can make the patient management more complex, however, being not an absolute contraindication for a VA creation.

Probably, the "low compliance" of a patient affected by geriatric syndrome and dementia represents the major obstacle to VA surgery, not allowing an LA procedure and conditioning deep sedation or GA.

\section{Is the old age a limiting factor for the VA management?}

HD access failure remains a major source of morbidity and it is second only to cardiovascular disease as a cause of hospitalization for ESRD patients: for this reason, a correct VA management is a crucial point to improve fistula and graft patency and to have low complication rates, both for young and old patients.

The daily care of the VA represents the mainstay in preservation and long-term "life" of the VA, and for elderly patients, its management may require more resources and the continuative cooperation between patient, relatives, nurses, nephrologists, vascular surgeons, and interventional radiologists.

This ideal condition is hardly established in small peripheral hospital, where the resources are lower and a multidisciplinary team is often missing; however, this obstacle can be overcome if the peripheral hospital can refer to central structure with an available "access management team," as in a "spoke-to-hub" model and especially with a "VA culture" spreading. This starts from the patient itself, if collaborative, passing through family members, dialysis nurses, and family doctors, who can allow a correct follow-up program focused on recognizing early signs of VA malfunction. ${ }^{30,31}$

\section{Declaration of conflicting interests}

The author(s) declared no potential conflicts of interest with respect to the research, authorship, and/or publication of this article.

\section{Funding}

The author(s) received no financial support for the research, authorship, and/or publication of this article.

\section{References}

1. Frailty in older people. Lancet 2013; 381(9868): 752-762.

2. Mallappalli M and Friedman E. Chronic kidney disease in the elderly: evaluation and management. Clin Pract 2014; 11(5): 525-535.

3. Dousdampanis P, Trigka K and Fourtounas C. Diagnosis and management of chronic kidney disease in the elderly: a field of ongoing debate. Aging Dis 2012; 3(5): 360372. 
4. Dimkovic N. Management of elderly patients with endstage kidney disease. Semin Nephrol 2009 29(6): 643-649.

5. Brunori CG, Viola G, Parrinello BF, et al. Efficacy and safety of a very-low-protein diet when postponing dialysis in the elderly: a prospective randomized multicenter controlled study. Am J Kidney Dis 2007; 49(5): 569-580.

6. Anand S, Kurella Tamura M and Chertow GM. The elderly patients on hemodialysis. Minerva Urol Nefrol 2010; 62(1): 87-101.

7. Hall RK, Myers ER, Rosas SE, et al. Choice of hemodialysis access in older adults: a cost-effectiveness analysis. Clin J Am Soc Nephrol 2017; 12(6): 947-954.

8. Bray BD, Boyd J, Daly C, et al. Vascular access type and risk of mortality in a national prospective cohort of haemodialysis patients. QJM 2012; 105: 1097-1102.

9. Aitken E, Geddes C, Thomson P, et al. Immediate access arteriovenous grafts versus tunnelled central venous catheters: study protocol for a randomised controlled trial. Trials 2015; 16(1): 42.

10. Woo K and Lok C. New insights into dialysis vascular access: what is the optimal vascular access type and timing of access creation in CKD and dialysis patients? Clin J Am Soc Nephrol 2016; 11: 1487-1494.

11. Wasse H, Speckman RA, Frankenfield DL, et al. Predictors of delayed transition from central venous catheter use to permanent vascular access among ESRD patients. Am $J$ Kidney Dis 2007; 49: 276-283.

12. Tordoir J, Canaud B, Haage P, et al. EBPG on vascular access. Nephrol Dial Transplant 2007; 22(Suppl. 2): 88117.

13. Macfarlane AJR, Kearns R, Aitken E, et al. Does regional compared to local anaesthesia influence outcome after arteriovenous fistula creation? Trials 2013; 14(1): 263.

14. Tordoir JHM, Bode AS, van Loon MM, et al. Preferred strategy for hemodialysis access creation in elderly patients. Eur J Vasc Endovasc Surg 2015; 49(6): 738-743.

15. Sidawy AN, Spergel LM, Besarab A, et al. The society for vascular surgery: clinical practice guidelines for the surgical placement maintenance of arteriovenous hemodialysis access. J Vasc Surg 2008; 48: 2S-25S.

16. Allon $\mathrm{M}$ and Robbin $\mathrm{M}$. Increasing arteriovenous fistulas in hemodialysis patients: problem and solutions. Kidney Int 2002; 62: 1109-1124.

17. Lok C and Allon M. Risk equation determining unsuccessful cannulation events and failure to maturation in arteriovenous fistulas. J Am Soc Nephrol 2006; 17: 3204-3212.
18. Swindlehurst $\mathrm{N}$ and Swindlehurst A. Vascular access for hemodialysis in the elderly. J Vasc Surg 2011; 53: 10391043.

19. Olsha O and Hijazi J. Vascular access in hemodialysis patients older than 80 years. J Vasc Surg 2015; 61: 177-183.

20. DeSilva RN, Patibandla BK, Vin Y, et al. Fistula first is not always the best strategy for the elderly. J Am Soc Nephrol 2013; 24(8): 1297-1304.

21. Cui J, Steele D, Wenger J, et al. Hemodialysis arteriovenous fistula as first option not necessary in elderly patients. J Vasc Surg 2016; 63(5): 1326-1332.

22. Tamura MK. Incidence, management, and outcomes of end-stage renal disease in the elderly. Curr Opin Nephrol Hypertens 2009; 18(3): 252-257.

23. Tozzi M and Franchin M. Initial experience with the Gore ${ }^{\circledR}$ Acuseal graft for prosthetic vascular access. $J$ Vasc Access 2014; 15 : 385-390.

24. Aitken E, Thomson P, Bainbridge L, et al. A randomized controlled trial and cost-effectiveness analysis of early cannulation arteriovenous grafts versus tunneled central venous catheters in patients requiring urgent vascular access for hemodialysis. J Vasc Surg 2017; 65(3): 766-774.

25. Smith GE, Gohil R and Chetter IC. Factors affecting the patency of arteriovenous fistulas for dialysis access. $J$ Vasc Surg 2012; 55(3): 849-855.

26. Malovrh M. Native arteriovenous fistula: preoperative evaluation. Am J Kidney Dis 2002; 39(6): 1218-1225.

27. Kordzadeh A, Chung J and Panayiotopoulos YP. Cephalic vein and radial artery diameter in formation of radiocephalic arteriovenous fistula: a systematic review. J Vasc Access 2015; 16(6): 506-511.

28. Lomonte C, Forneris G, Gallieni M, et al. The vascular access in the elderly: a position statement of the Vascular Access Working Group of the Italian Society of Nephrology. J Nephrol 2016; 29(2): 175-184.

29. Zamani P, Kaufman J and Kinlay S. Ischemic steal syndrome following arm arteriovenous fistula for hemodialysis. Vasc Med 2009; 14(4): 371-376.

30. Mishler R, Sands JJ, Ofsthun NJ, et al. Dedicated outpatient vascular access center decreases hospitalization and missed outpatient dialysis treatments. Kidney Int 2006; 69(2): 393 398.

31. Beathard GA. Role of interventional nephrology in the multidisciplinary approach to hemodialysis vascular access care. Kidney Res Clin Pract 2015; 34(3): 125-131. 